\title{
Red meat intake is positively associated with non-fatal acute myocardial infarction in the Costa Rica Heart Study
}

\author{
Dongqing Wang ${ }^{1}$, Hannia Campos ${ }^{2,3}$ and Ana Baylin ${ }^{1,4 *}$ \\ ${ }^{1}$ Department of Epidemiology, School of Public Health, University of Michigan, Ann Arbor, MI 48109, USA \\ ${ }^{2}$ Department of Nutrition, Harvard T.H. Chan School of Public Health, Boston, MA 02115, USA \\ ${ }^{3}$ Centro de Investigación e Innovación en Nutricion Traslacional y Salud, Universidad Hispanoamericana, San Jose, Costa Rica \\ ${ }^{4}$ Department of Nutritional Sciences, School of Public Health, University of Michigan, Ann Arbor, MI 48109, USA
}

(Submitted 17 April 2017 - Submitted 5 July 2017 - Accepted 11 July 2017)

\section{Abstract}

The adverse effect of red meat consumption on the risk for CVD is a major population health concern, especially in developing Hispanic/ Latino countries in which there are clear trends towards increased consumption. This population-based case-control study examined the associations between total, processed and unprocessed red meat intakes and non-fatal acute myocardial infarction (MI) in Costa Rica. The study included 2131 survivors of a first non-fatal acute MI and 2131 controls individually matched by age, sex and area of residence. Dietary intake was assessed with a FFQ. OR were estimated by using conditional logistic regression. Higher intakes of total and processed red meat were associated with increased odds of acute MI. The OR were 1.31 (95\% CI 1.04, 1.65) and 1.29 (95\% CI 1.01, 1.65) for the highest quintiles of total red meat (median: $110 \cdot 8 \mathrm{~g}$ or 1 serving/d) and processed red meat intake (median: $36.1 \mathrm{~g}$ or 5 servings/week), respectively. There were increasing trends in the odds of acute MI with higher total $\left(P_{\text {trend }}=0.01\right)$ and processed $\left(P_{\text {trend }}=0.02\right)$ red meat intakes. Unprocessed red meat intake was not associated with increased odds of acute MI. Substitutions of $50 \mathrm{~g}$ of alternative foods (fish, milk, chicken without skin and chicken without fat) for $50 \mathrm{~g}$ of total, processed and unprocessed red meat were associated with lower odds of acute MI. The positive association between red meat intake and acute MI in Costa Rica highlights the importance of reducing red meat consumption in middleincome Hispanic/Latino populations.

\section{Key words: Red meat: Processed meat: CVD: Myocardial infarction: Costa Rica}

Red meat consumption has notably increased in developing countries over the last 50 years $^{(1,2)}$ and will continue to do so moving forward. It is estimated that between 2017 and 2023, developing countries will account for $83 \%$ of the increase in meat consumption relative to $2010-2013^{(3)}$. These trends are worrisome given the potential adverse effects of red meat intake on CVD, the main cause of death in developing countries which also currently represent $80 \%$ of the global CVD burden ${ }^{(4-6)}$.

Epidemiological studies have suggested that high intake of red meat, especially processed red meat, is associated with some major chronic diseases $^{(7)}$. Red meat intake is associated with higher CVD incidence and mortality in studies conducted in high-income countries including the USA and some European countries ${ }^{(8-10)}$. In the USA, it is estimated that increasing processed and unprocessed red meat intake by 1 serving/d is associated with 21 and $18 \%$ higher risk of CVD mortality, respectively ${ }^{(8)}$.

To the best of our knowledge, studies on the effect of red meat intake on CVD in Hispanic/Latino populations are extremely scarce. Hispanic/Latino countries have been transitioning to a more Westernised diet ${ }^{(11)}$. It is possible that the increase in CVD incidence in developing Hispanic/Latino countries is partially due to the increase in red meat consumption due to this shift toward a more Westernised diet.

The purpose of our study is to investigate the association between intakes of total, processed and unprocessed red meat and non-fatal acute myocardial infarction (MI), a form of CVD, in Costa Rica, a developing Hispanic/Latino country. In Costa Rica, CVD is the most common cause of death, estimated to account for $30 \%$ of total deaths ${ }^{(12)}$. We also evaluated potential effect modifiers and the benefits of substituting other alternative sources of protein for total, processed and unprocessed red meat. We hypothesised that intake of red meat, especially processed red meat, would be positively associated with acute MI in Costa Rica.

\section{Methods}

\section{Subjects}

All subjects were participants of the Costa Rica Heart Study, a population-based case-control study of diet and risk of CVD.

Abbreviation: MI, myocardial infarction.

* Corresponding author: A. Baylin, fax +1 734764 3192, email abaylin@umich.edu 
All subjects were Hispanics/Latinos who lived in the Central Valley of Costa Rica between 1994 and 2004. The catchment area for the study included thirty-four counties and covered a full range of lifestyles and socioeconomic characteristics. The details of the study have been described elsewhere ${ }^{(13)}$. In brief, eligible cases were adult residents (men and women) who were diagnosed as survivors of a first acute MI by independent examinations of two cardiologists at any of the six recruiting hospitals in the catchment area. All cases met the WHO criteria for MI, which includes typical symptoms as well as either elevations in cardiac enzyme concentrations or diagnostic changes in the electrocardiogram ${ }^{(14)}$. One free-living population-based control subject for each case, matched for age ( \pm 5 years), sex and area of residence (county), was randomly selected with the aid of the information available at the National Census and Statistics Bureau of Costa Rica. Because of the comprehensive social services provided in Costa Rica, all persons living in the catchment area had access to medical care regardless of their income level. Therefore, control subjects came from the source population that gave rise to the cases and were unlikely to have had undiagnosed CVD because of limited access to health care. Participation was $98 \%$ for cases and $88 \%$ for controls. All subjects gave informed consent on documents approved by the Human Subjects Committee of the Harvard School of Public Health and the University of Costa Rica.

The initial study population consisted of 2273 case-control pairs ( $n$ 4546). We excluded participants who had missing information on red meat intake ( $n$ 118) or major confounders (waist:hip ratio, physical activity level, monthly household income, history of diabetes, history of hypertension, smoking status, alcohol intake, total energy intake, fruit servings, green leafy vegetable servings, chicken intake and fish intake; $n$ 43), and who were unable to be re-matched after the aforementioned exclusions ( $n$ 123). Data from 2131 case-control pairs were available for data analysis (Fig. 1).

\section{Data collection}

On average, data collection for both cases and controls took place 3 weeks after hospital discharge of the case subject. Trained personnel visited study participants at their homes. Data on demographic characteristics, cigarette smoking, alcohol intake, physical activity, socioeconomic status and medical history were collected using a questionnaire with close-ended questions. Anthropometric and blood pressure measurements were also taken. To calculate the physical activity level, we multiplied the frequency, duration and intensity of each physical activity level, and then summed up the energy expenditures from all activities to derive total metabolic equivalents, as described earlier ${ }^{(15)}$. All participants were also asked to choose from a list of factors which they believed was 'the main cause of heart attacks'. Possible responses included diet, obesity, smoking, alcohol intake, coffee intake, physical activity, stress, occupation, medication, sexual activity, genetic factors, high blood cholesterol, high blood glucose and high blood pressure.

We measured dietary intake using a 135 -item semi-quantitative FFQ that was developed and validated specifically to assess food and nutrient intake in the Costa Rican population ${ }^{(16,17)}$. This FFQ was based on the Willett questionnaire ${ }^{(18)}$, and we added specific items from the Costa Rican diet ${ }^{(19)}$, which was a typical Central American diet based on rice, beans, plantains, maize tortillas, small salads with chopped vegetables, meat, eggs and cheese. Intake of energy and nutrients was computed by multiplying the consumption frequency of each food by the nutrient content of the specific portion. We used food composition values from the US Department of Agriculture database and data from manufacturers and published reports ${ }^{(18,20)}$. In a validation study among 120 randomly selected control subjects, we used the method of triads to validate the FFQ against 7-d, 24-h dietary recalls, and biomarkers of fatty acids, tocopherols and carotenoids in

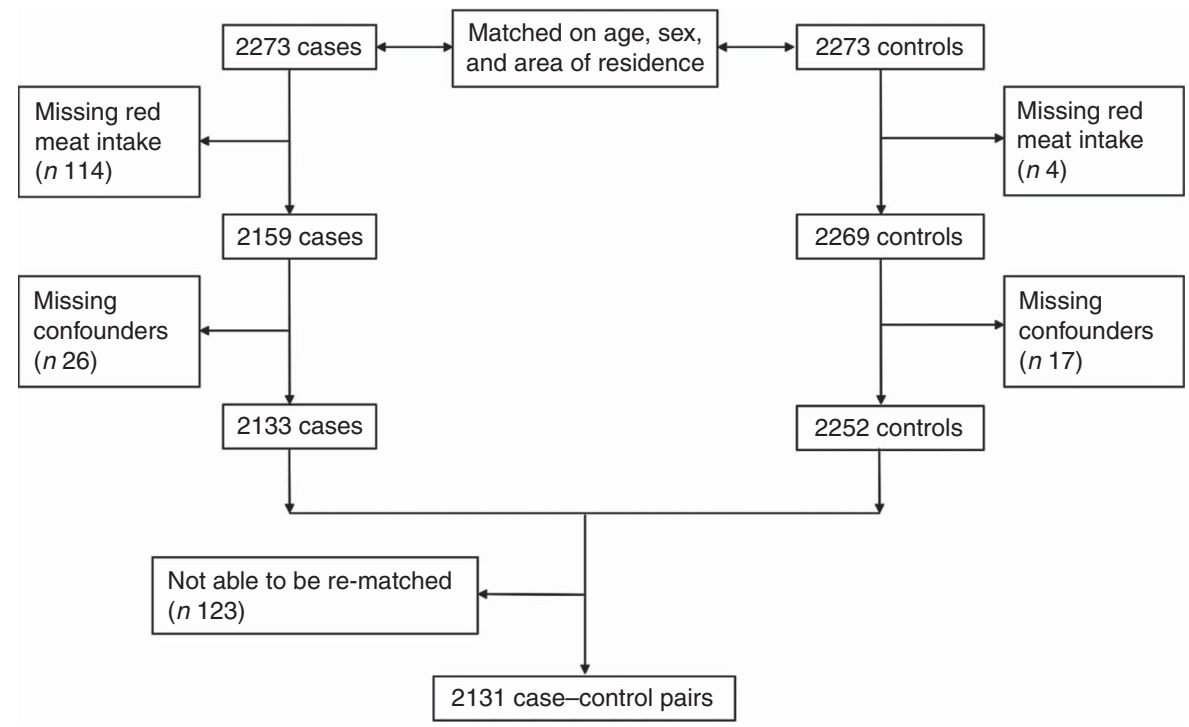

Fig. 1. The flow of participants through the Costa Rica Heart Study. We excluded participants who did not have any data on the processed and unprocessed red meat items. After this exclusion, fifteen participants had remaining missing red meat intake items, the values of which were subsequently set to 0 . The confounders included waist:hip ratio, physical activity level, monthly household income, history of diabetes, history of hypertension, smoking status, alcohol intake, total energy intake, fruit servings, green leafy vegetable servings, chicken intake and fish intake. 
plasma and adipose tissue ${ }^{(16)}$. We also compared the FFQ against a second FFQ administered 1 year apart to assess reproducibility ${ }^{(16)}$. The Pearson partial correlation coefficients for total fatty acid, SFA, MUFA and PUFA between the averages of the two FFQ assessments and the 7-d, 24-h recalls were 0.74, 0.71, 0.64 and 0.75, respectively; the values were $0.46,0 \cdot 60,0.47$ and 0.59 , respectively, between the first and second FFQ ( $P<0 \cdot 0001$ for all values). The FFQ was administered by trained personnel. The participants were asked how often, on average, they consumed each food of a standard portion size during the past year. For most foods, there were nine possible responses for frequency: never or $<1$ time/ month, 1-3 times/month, 1 time/week, 2-4 times/week, 5-6 times/ week, 1 time/d, 2-3 times/d, 4-5 times/d and $\geq 6$ times/d.

\section{Definitions}

For the purpose of our study, 'red meat' was defined as meat from mammals such as cows, sheep, veal calves, lamb and pigs. 'Processed red meat' referred to red meat that has been preserved by smoking, salting or addition of chemical preservatives, such as bacon, salami, sausages or hot dogs. 'Unprocessed red meat' was defined as fresh red meat free from these preservation methods. Processed and unprocessed red meat contain the similar amount of SFA and haem $\mathrm{Fe}^{(8)}$, whereas they differ most notably in their $\mathrm{Na}$ and nitrate/nitrite contents because of the food preservation methods used for processed red meat ${ }^{(21)}$. FFQ items on unprocessed red meat intake included 'hamburger', 'beef, pork or lamb as a main dish', 'beef, pork or lamb as a sandwich' and 'beef, pork or lamb in mixed dishes'. Processed red meat items included 'bacon', 'hot dogs' and 'sausage, bologna, salami, ham and other processed meats'. The online Supplementary Table S1 provides more details of the compositions of processed red meat, unprocessed red meat, chicken and fish groups used in our study, as well as the standard serving sizes for each food item. For the interpretation of our results, we used $100 \mathrm{~g}$ as the average serving size for total red meat and unprocessed red meat and used $50 \mathrm{~g}$ as the average serving size for processed red meat ${ }^{(22,23)}$.

\section{Statistical methods}

We transformed the frequencies of food intake from the FFQ to semi-continuous variables representing the numbers of standard servings per day, with values of $0,0 \cdot 08,0 \cdot 14,0 \cdot 43,0 \cdot 8$, $1,2.5,4.5$ and 6.0 representing the nine possible responses in the FFQ respectively. We then multiplied the number of servings per day and the standard portion size of each food to derive the continuous weighted dietary variable (in g). We further adjusted all weighted dietary variables for total energy intake by using the residual method ${ }^{(24)}$ and used the energy-adjusted dietary variables in the subsequent models.

We assessed the significance of differences in general characteristics and dietary intake between cases and controls by using paired $t$ tests for normally distributed continuous variables, Wilcoxon's signed-rank tests for skewed continuous variables, and McNemar's tests for categorical variables. We examined the distributions of potential confounders among controls by quintiles of the energy-adjusted total, processed and unprocessed red meat intakes (online Supplementary Table S2). OR and $95 \%$ CI of acute MI associated with total, processed and unprocessed red meat intakes were estimated by using conditional logistic regression models. We used quintile-based categorisation of the exposure variables instead of the original continuous variables because dose-response relationships and potential non-linear associations could be evaluated. In addition, we computed the $P_{\text {trend }}$ across quintiles of red meat intakes by assigning the median intake value of each quintile to the subjects in the corresponding quintile as a continuous variable in the model. Non-dietary confounders included in the final multivariate models were waist:hip ratio (quintiles), physical activity level (quintiles), monthly household income (quintiles plus an indicator for missing data), history of diabetes (yes or no), history of hypertension (yes or no), smoking status (never, former or current $<10,10-20$ or $>20$ cigarettes/d), and alcohol intake (never, former or current tertiles). Dietary confounders included total energy intake, fruit servings, green leafy vegetable servings, energy-adjusted chicken intake and energy-adjusted fish intake (all in quintiles). We also examined models in which processed and unprocessed red meat intake (both energy-adjusted) were adjusted for each other. Adjustments of formal education level and type of oil used did not appreciably change the results, so were not included in the final models as covariates. The multiplicative interaction between processed/unprocessed red meat intake and potential effect modifiers (age, sex, waist:hip ratio and physical activity level) was tested by including product terms in the fullest multivariate model. The product terms were created using the median intake of each quintile (as a continuous variable) and each potential effect modifier (sex as a binary variable; age, waist:hip ratio and physical activity level as continuous variables). As heterogeneous results by sex have been reported by some studies ${ }^{(4)}$, we also conducted sex-stratified analysis to further evaluate effect modification by sex.

The associations of substituting $50 \mathrm{~g}$ of an alternative source of protein for $50 \mathrm{~g}$ of total, processed and unprocessed red meat with acute MI were estimated by including the numbers of servings (standardised to $50 \mathrm{~g}$ ) of both foods as continuous variables in the same model, which also included all non-dietary confounders plus total energy intake ${ }^{(8)}$. The difference in the two $\beta$-coefficients, as well as their variances and covariance, were used to estimate the OR and its 95\% CI associated with the substitution of interest ${ }^{(25)}$. The alternative foods examined included fish, chicken with skin and fat, chicken without skin or chicken without fat, whole milk and low-fat milk (including 1 and $2 \%$ milk), which were all potentially healthier food sources of protein $^{(8,25)}$. The substitution analysis made the assumption that the associations between the alternative foods and acute MI were roughly linear on the log odds scale. We evaluated this assumption by examining the associations between the quintiles of each alternative food and acute MI, adjusted for all non-dietary confounders and total energy intake. We did not find any notable deviation from linearity (e.g. no U-shape pattern was found between any alternative food and acute MI).

To examine the robustness of the results, we performed five sensitivity analyses. First, we conducted multiple imputations on the missing data of monthly household income ( $n 315 ; 7.4 \%$ of the entire sample). A multiple Markov chain Monte Carlo method was used assuming missing at random ${ }^{(26)}$. Variables used to predict missing income data included age, sex, area of residence, 
physical activity level, formal education level, alcohol intake, green leafy vegetable servings and case-control status of acute MI. Second, we repeated the analyses after excluding participants with missing income (i.e. a complete case analysis). Third, we repeated the analyses after excluding participants who reported implausible total energy intake $(<2092 \mathrm{~kJ} / \mathrm{d}(<500 \mathrm{kcal} / \mathrm{d})$ or $>14644 \mathrm{~kJ} / \mathrm{d}(>3500 \mathrm{kcal} / \mathrm{d}) ; n$ 529). Fourth, to address potential differential exposure measurement error, we repeated the analyses excluding participants who believed that diet was the main cause of MI ( $n$ 621). Last, as the history of hypertension could be a mediator instead of a confounder, we repeated the analysis without adjusting for the history of hypertension in the fullest models.

A power calculation was performed using data from a prospective investigation of red meat intake and the risk of CHD in the Nurses' Health Study ${ }^{(25)}$, in which an OR of 1.29 was found comparing the highest to lowest quintiles of total red meat intake. Under this estimated effect size and a two-sided significance level of 0.05 , our study ( $n$ 4262) had a statistical power of $93 \%$. All data were analysed by using the SAS software, version 9.4 (SAS Institute Inc.).

\section{Results}

Most of the examined general characteristics and dietary factors were significantly different between cases and controls in the expected direction, except for MUFA, trans-fat, chicken intake, fruit servings and green leafy vegetable servings (Table 1). Compared with controls, cases had higher intakes of energyadjusted total, processed and unprocessed red meat intakes, as well as lower energy-adjusted fish intake. Energy-adjusted intakes of processed and unprocessed red meat were weakly and positively correlated (Pearson's $r 0 \cdot 16$ and $0 \cdot 21$ in cases and controls, respectively).

Higher intake of total and processed red meat intakes were associated with higher odds of acute MI after adjusting for potential confounders (Table 2). Compared with participants in the lowest quintile of total red meat intake (energy-adjusted median intake: $19.5 \mathrm{~g}$ or 1.5 servings/week), those in the highest quintile (energy-adjusted median intake: $110.8 \mathrm{~g}$ or 1 serving/d) has higher odds of acute MI (OR 1.31; $95 \%$ CI 1.04, 1.65). There was also a significant increasing trend in the odds of acute MI with higher total red meat intake $\left(P_{\text {trend }}=0 \cdot 01\right)$, though the second to the fourth quintiles of total red meat intake were not significantly associated with the odds of acute MI. Analyses that evaluated processed and unprocessed meat independently show that, compared with participants in the lowest quintile of processed red meat intake (energy-adjusted median intake: $0 \mathrm{~g}$ ), the highest quintile of processed red meat intake (energy-adjusted median intake: $36 \cdot 1 \mathrm{~g}$ or 5 servings/ week) was associated with higher risk of acute MI (OR 1.29; $95 \%$ CI 1.01, 1.65). The association between the highest quintile of unprocessed red meat intake and the odds of acute MI was not statistically significant.

In the sex-stratified analysis, the associations were notably stronger in women than in men. Among women, the OR for the highest quintile of total, processed and unprocessed red meat intake were 1.57 (95\% CI 0.93, 2.64), 1.47 (95\% CI 0.80, 2.69) and
Table 1. General characteristics and dietary factors of the 2131 cases of non-fatal acute myocardial infarction and the 2131 population-based matched controls in the Costa Rica Heart Study

(Mean values and standard deviations; percentages)

\begin{tabular}{|c|c|c|c|c|}
\hline & \multicolumn{2}{|c|}{$\begin{array}{l}\text { Controls } \\
(n 2131)\end{array}$} & \multicolumn{2}{|c|}{$\begin{array}{l}\text { Cases } \\
(n 2131) \\
\end{array}$} \\
\hline & Mean & SD & Mean & SD \\
\hline \multicolumn{5}{|l|}{ Matching variables } \\
\hline Age (years) & $58 \cdot 1$ & 11.3 & 58.5 & $11 \cdot 1$ \\
\hline Women $(\%)$ & \multicolumn{2}{|c|}{$26 \cdot 6$} & \multicolumn{2}{|c|}{$26 \cdot 6$} \\
\hline Residence in urban areas (\%) & \multicolumn{2}{|c|}{38.8} & \multicolumn{2}{|c|}{38.8} \\
\hline \multicolumn{5}{|l|}{ General characteristics } \\
\hline 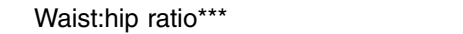 & 0.95 & 0.08 & 0.97 & 0.07 \\
\hline Physical activity $(\mathrm{MET} / \mathrm{d})^{\star *}$ & 35.5 & $16 \cdot 2$ & 34.4 & $16 \cdot 1$ \\
\hline $\begin{array}{l}\text { Monthly household income } \\
\text { (US dollars) } \dagger^{\star \star \star}\end{array}$ & 571.6 & $426 \cdot 3$ & $496 \cdot 4$ & 391.4 \\
\hline Secondary education or higher $(\%)^{*}$ & \multicolumn{2}{|c|}{$40 \cdot 1$} & \multicolumn{2}{|c|}{$36 \cdot 7$} \\
\hline History of diabetes $(\%)^{\star \star \star}$ & \multirow{2}{*}{\multicolumn{2}{|c|}{$\begin{array}{l}14.1 \\
29.3\end{array}$}} & \multicolumn{2}{|c|}{$24 \cdot 6$} \\
\hline History of hypertension $(\%)^{\star \star \star}$ & \multirow{2}{*}{\multicolumn{2}{|c|}{$\begin{array}{l}29.3 \\
21.1\end{array}$}} & \multicolumn{2}{|c|}{38.5} \\
\hline Current smoker $(\%)^{\star \star \star}$ & & & \multirow{2}{*}{\multicolumn{2}{|c|}{$\begin{array}{l}39.9 \\
48.7\end{array}$}} \\
\hline Current alcohol drinker $(\%)^{\star \star}$ & \multicolumn{2}{|c|}{$52 \cdot 9$} & & \\
\hline \multicolumn{5}{|l|}{ Dietary intake } \\
\hline Total energy intake $(\mathrm{MJ} / \mathrm{d})^{\star \star \star}$ & $10 \cdot 2$ & $3 \cdot 2$ & $11 \cdot 3$ & 3.9 \\
\hline Total fat $(\% \text { energy })^{\star *}$ & 31.9 & 5.9 & 32.4 & 5.9 \\
\hline SFA $(\% \text { energy })^{\star \star \star}$ & $11 \cdot 7$ & $2 \cdot 9$ & $12 \cdot 5$ & $3 \cdot 1$ \\
\hline MUFA (\% energy) & $11 \cdot 2$ & 4.0 & $11 \cdot 2$ & 3.5 \\
\hline PUFA (\% energy) ${ }^{\star *}$ & $7 \cdot 1$ & $2 \cdot 3$ & $6 \cdot 8$ & $2 \cdot 3$ \\
\hline Trans-fat (\% energy) & 1.3 & 0.6 & 1.3 & 0.6 \\
\hline Carbohydrates (\% energy) ${ }^{\star \star \star}$ & 55.4 & $7 \cdot 3$ & 54.4 & 7.5 \\
\hline Protein (\% energy) $)^{\star \star \star}$ & $12 \cdot 9$ & $2 \cdot 1$ & $13 \cdot 2$ & $2 \cdot 2$ \\
\hline Cholesterol $(\mathrm{mg} / \mathrm{kJ})^{\star * \star}$ & $28 \cdot 1$ & $12 \cdot 4$ & 30.2 & 14.0 \\
\hline $\mathrm{Na}(\mathrm{g} / \mathrm{d}) \ddagger^{*}$ & $2 \cdot 28$ & 0.45 & 2.31 & 0.53 \\
\hline Total red meat $(\mathrm{g} / \mathrm{d}) \ddagger^{\star \star \star}$ & $60 \cdot 8$ & $36 \cdot 8$ & $66 \cdot 7$ & $46 \cdot 1$ \\
\hline Processed red meat $(\mathrm{g} / \mathrm{d}) \ddagger^{*}$ & $16 \cdot 0$ & $17 \cdot 0$ & $19 \cdot 0$ & $26 \cdot 2$ \\
\hline Unprocessed red meat $(\mathrm{g} / \mathrm{d}) \ddagger^{*}$ & 44.8 & 29.8 & $47 \cdot 8$ & 36.7 \\
\hline Fish $(g / d) \ddagger^{* \star *}$ & $30 \cdot 6$ & $22 \cdot 7$ & 28.8 & 24.9 \\
\hline Chicken $(\mathrm{g} / \mathrm{d}) \ddagger$ & $35 \cdot 7$ & $25 \cdot 5$ & 37.4 & 29.4 \\
\hline Fruits (servings/d)§ & 4.3 & 3.0 & $4 \cdot 2$ & $2 \cdot 7$ \\
\hline Green leafy vegetables (servings/d)\| & 0.8 & 0.6 & 0.8 & 0.6 \\
\hline
\end{tabular}

Mean values or percentages were significantly different between cases and controls: ${ }^{\star} P<0.05,{ }^{\star *} P<0.01,{ }^{\star \star \star} P<0.001$.

† There were 315 participants with missing monthly household income, resulting in 1972 controls and 1975 cases.

$\ddagger$ Adjusted for total energy intake using the residual method.

$\S$ Includes pineapples, papaya, bananas, cantaloupe, apples, pears, mango, oranges, watermelon, tangerine, lychee, peach palm, avocado, tomatoes, cukes, plantain, zucchini and yellow squash.

|| Includes broccoli, coleslaw, cabbage, spinach, kale and lettuce.

1.44 (95\% CI 0.86, 2.40), respectively (Table 3); among men, the values were 1.19 (95\% CI 0.92, 1.55), 1.25 (95\% CI 0.95, 1.65) and 1.01 (95\% CI $0.78,1 \cdot 32)$, respectively (Table 4 ). The associations were not statistically significant in neither men nor women, possibly due to the reduced power of the stratified analysis. However, based on the Wald $\chi^{2}$ test of the product term, there was a marginally significant interaction between sex and unprocessed red meat intake $(P=0.054)$ indicating that the effect of unprocessed red meat intake was stronger in women than in men. No statistical interactions were detected between total and processed red meat intake and sex $(P>0 \cdot 10$ for both product terms). We did not find significant interactions between total, processed or unprocessed red meat intakes and age, waist:hip ratio or physical activity level ( $P>0 \cdot 10$ for all product terms).

The estimates were largely unchanged when we did not adjust for history of hypertension in the fullest models. The OR for total red meat intake were 1.00 (reference), 1.02 (95\% CI 
Table 2. Quintiles $(Q)$ of total, processed and unprocessed red meat intakes and non-fatal acute myocardial infarction among the 2131 case-control pairs from the Costa Rica Heart Study

(Odds ratios and $95 \%$ confidence intervals)

\begin{tabular}{|c|c|c|c|c|c|c|c|c|c|c|}
\hline & \multirow[b]{2}{*}{ Q1 } & \multicolumn{2}{|c|}{ Q2 } & \multicolumn{2}{|c|}{ Q3 } & \multicolumn{2}{|c|}{ Q4 } & \multicolumn{2}{|c|}{ Q5 } & \multirow[b]{2}{*}{$P_{\text {trend }}$} \\
\hline & & OR & $95 \% \mathrm{Cl}$ & OR & $95 \% \mathrm{Cl}$ & OR & $95 \% \mathrm{Cl}$ & OR & $95 \% \mathrm{Cl}$ & \\
\hline \multicolumn{11}{|l|}{ Total red meat } \\
\hline Median intake $(\mathrm{g} / \mathrm{d})^{\star}$ & 19.5 & \multicolumn{2}{|c|}{$42 \cdot 6$} & \multicolumn{2}{|c|}{58.6} & \multicolumn{2}{|c|}{78.2} & \multicolumn{2}{|c|}{$110 \cdot 8$} & \\
\hline Basic model† & 1.00 & 0.83 & $0.69,1.01$ & 0.88 & $0.72,1.07$ & 0.92 & $0.76,1 \cdot 12$ & 1.39 & $1 \cdot 15,1.69$ & $<0.001$ \\
\hline Multivariate $1 \ddagger$ & 1.00 & 0.83 & $0.68,1.03$ & 0.82 & $0.66,1.02$ & 0.91 & $0.73,1.14$ & 1.27 & $1.02,1.58$ & 0.007 \\
\hline Multivariate $2 \S$ & 1.00 & 1.00 & $0.80,1.26$ & 1.04 & $0.82,1.31$ & 1.05 & $0.83,1.32$ & 1.31 & $1.04,1.65$ & 0.01 \\
\hline \multicolumn{11}{|l|}{ Processed red meat } \\
\hline Median intake $(\mathrm{g} / \mathrm{d})^{\star}$ & 0 & \multicolumn{2}{|c|}{8.4} & \multicolumn{2}{|c|}{$14 \cdot 3$} & \multicolumn{2}{|c|}{20.9} & \multicolumn{2}{|c|}{$36 \cdot 1$} & \\
\hline Basic model† & 1.00 & 0.79 & $0.65,0.96$ & 0.74 & $0.61,0.90$ & 0.78 & $0.65,0.95$ & $1 \cdot 16$ & $0.95,1.41$ & 0.05 \\
\hline Multivariate $1 \ddagger$ & 1.00 & 0.78 & $0.63,0.97$ & 0.73 & $0.59,0.92$ & 0.74 & $0.59,0.92$ & 1.08 & $0.86,1.35$ & 0.31 \\
\hline Multivariate $2 \S$ & 1.00 & 0.99 & $0.78,1.26$ & 1.04 & $0.81,1.34$ & 1.03 & $0.80,1.31$ & 1.32 & $1.04,1.68$ & 0.01 \\
\hline Multivariate 3\| & 1.00 & 0.98 & $0.77,1.25$ & 1.03 & $0.80,1.33$ & 1.01 & $0.79,1.30$ & 1.29 & $1.01,1.65$ & 0.02 \\
\hline \multicolumn{11}{|l|}{ Unprocessed red meat } \\
\hline Median intake $(\mathrm{g} / \mathrm{d})^{\star}$ & 11.5 & \multicolumn{2}{|c|}{28.0} & \multicolumn{2}{|c|}{$41 \cdot 1$} & \multicolumn{2}{|c|}{$58 \cdot 7$} & \multicolumn{2}{|c|}{85.6} & \\
\hline Basic model† & 1.00 & 0.88 & $0.73,1.07$ & 0.82 & $0.68,1.00$ & 0.99 & $0.81,1.19$ & 1.22 & $1.01,1.49$ & 0.006 \\
\hline Multivariate $1 \ddagger$ & 1.00 & 0.90 & $0.73,1.11$ & 0.81 & $0.65,1.01$ & 0.95 & $0.77,1.18$ & 1.16 & $0.93,1.44$ & 0.07 \\
\hline Multivariate $2 \S$ & 1.00 & $1 \cdot 10$ & $0.88,1.38$ & 0.98 & $0.78,1.24$ & 1.02 & $0.81,1.28$ & 1.18 & $0.94,1.47$ & 0.25 \\
\hline Multivariate 39 & 1.00 & 1.08 & $0.86,1.36$ & 0.96 & $0.76,1.22$ & 1.00 & $0.79,1.26$ & 1.13 & $0.90,1.42$ & 0.42 \\
\hline
\end{tabular}

* Adjusted for total energy intake using the residual method.

$\dagger$ Adjusted for the matching factors (age, sex and area of residence).

$\ddagger$ In addition to the basic model, adjusted for waist:hip ratio (quintiles), physical activity level (quintiles), monthly household income (quintiles plus an indicator for missing data), history of diabetes (yes or no), history of hypertension (yes or no), smoking status (never, former or current $<10,10-20$ or $>20$ cigarettes/d) and alcohol intake (never, former or current tertiles).

$\S$ In addition to the multivariate 1 , adjusted for total energy intake, fruit servings, green leafy vegetable servings, energy-adjusted chicken intake and energy-adjusted fish intake (all in quintiles).

\|I In addition to the multivariate 2, adjusted for energy-adjusted unprocessed red meat intake in quintiles (mutual adjustment).

II In addition to the multivariate 2, adjusted for energy-adjusted processed red meat intake in quintiles (mutual adjustment).

Table 3. Quintiles $(Q)$ of total, processed and unprocessed red meat intakes and non-fatal acute myocardial infarction among 1132 women from the Costa Rica Heart Study

(Odds ratios and $95 \%$ confidence intervals)

\begin{tabular}{|c|c|c|c|c|c|c|c|c|c|c|}
\hline & \multirow[b]{2}{*}{ Q1 } & \multicolumn{2}{|c|}{ Q2 } & \multicolumn{2}{|c|}{ Q3 } & \multicolumn{2}{|c|}{ Q4 } & \multicolumn{2}{|c|}{ Q5 } & \multirow[b]{2}{*}{$P_{\text {trend }}$} \\
\hline & & OR & $95 \% \mathrm{Cl}$ & OR & $95 \% \mathrm{Cl}$ & OR & $95 \% \mathrm{Cl}$ & OR & $95 \% \mathrm{Cl}$ & \\
\hline \multicolumn{11}{|l|}{ Total red meat } \\
\hline Basic model ${ }^{\star}$ & 1.00 & 1.05 & $0.74,1.50$ & 1.09 & $0.77,1.55$ & $1 \cdot 30$ & $0.88,1.90$ & 1.71 & $1.14,2.57$ & 0.005 \\
\hline Multivariate $1 \dagger$ & 1.00 & 1.02 & $0.69,1.53$ & 0.88 & $0.59,1.32$ & 1.20 & $0.76,1.88$ & 1.42 & $0.88,2 \cdot 30$ & 0.12 \\
\hline Multivariate $2 \ddagger$ & 1.00 & 1.36 & $0.87,2 \cdot 14$ & $1 \cdot 14$ & $0.72,1.81$ & 1.49 & $0.91,2.44$ & 1.57 & $0.93,2.64$ & $0 \cdot 10$ \\
\hline \multicolumn{11}{|c|}{ Processed red meat } \\
\hline Basic model ${ }^{\star}$ & 1.00 & 0.99 & $0.68,1.44$ & 0.86 & $0.59,1.25$ & 0.77 & $0.53,1.12$ & 1.34 & $0.87,2.04$ & 0.48 \\
\hline Multivariate $1 \dagger$ & 1.00 & 0.82 & $0.53,1.28$ & 0.73 & $0.47,1.15$ & 0.63 & $0.40,0.99$ & 1.09 & $0.66,1.81$ & 0.85 \\
\hline Multivariate $2 \ddagger$ & 1.00 & 1.36 & $0.79,2.35$ & $1 \cdot 20$ & $0.68,2 \cdot 13$ & 1.07 & $0.61,1.88$ & 1.57 & $0 \cdot 86,2 \cdot 86$ & 0.29 \\
\hline Multivariate $3 \S$ & 1.00 & 1.30 & $0.75,2 \cdot 27$ & $1 \cdot 19$ & $0.67,2 \cdot 13$ & 1.02 & $0.57,1.81$ & 1.47 & $0.80,2.69$ & 0.41 \\
\hline \multicolumn{11}{|c|}{ Unprocessed red meat } \\
\hline Basic model* & 1.00 & 0.74 & $0.52,1.07$ & 0.83 & $0.57,1.21$ & 0.99 & $0.67,1.46$ & 1.57 & $1.04,2.35$ & 0.004 \\
\hline Multivariate $1 \dagger$ & 1.00 & 0.82 & $0.54,1.24$ & 0.82 & $0.53,1.28$ & 0.92 & $0.58,1.44$ & 1.43 & $0.89,2 \cdot 29$ & 0.08 \\
\hline Multivariate $2 \ddagger$ & 1.00 & 0.98 & $0.62,1.54$ & 1.03 & $0.63,1.68$ & 0.98 & $0.60,1.59$ & 1.49 & $0.90,2.46$ & $0 \cdot 12$ \\
\hline Multivariate $3 \|$ & 1.00 & 0.96 & $0.61,1.52$ & 1.01 & $0.62,1.66$ & 0.97 & $0.59,1.58$ & 1.44 & $0.86,2.40$ & 0.17 \\
\hline
\end{tabular}

* Adjusted for the matching factors (age and area of residence).

$\dagger$ In addition to the basic model, adjusted for waist:hip ratio (quintiles), physical activity level (quintiles), monthly household income (quintiles plus an indicator for missing data), history of diabetes (yes or no), history of hypertension (yes or no), smoking status (never, former or current $<10,10-20$ or $>20$ cigarettes/d) and alcohol intake (never, former or current tertiles).

$\mp$ In addition to the multivariate 1 , adjusted for total energy intake, fruit servings, green leafy vegetable servings, energy-adjusted chicken intake and energy-adjusted fish intake (all in quintiles).

$\S$ In addition to the multivariate 2, adjusted for energy-adjusted unprocessed red meat intake in quintiles (mutual adjustment).

|| In addition to the multivariate 2, adjusted for energy-adjusted processed red meat intake in quintiles (mutual adjustment).

$0 \cdot 82,1.28), 1.06$ (95\% CI $0 \cdot 84,1.33), 1.05$ (95\% CI $0 \cdot 83,1.32)$

and 1.31 (95\% CI 1.04, 1.64), for the lowest to highest quintiles respectively $\left(P_{\text {trend }}=0.02\right)$. The OR for processed red meat intake were 1.00 (reference), 0.98 (95\% CI $0.77,1.24), 1.02$ (95\% CI $0.79,1.31), 0.99$ (95\% CI $0.77,1.27)$ and 1.27 (95\% CI
$0.99,1.61)$, for the lowest to highest quintiles, respectively $\left(P_{\text {trend }}=0 \cdot 03\right)$. The OR for unprocessed red meat intake were 1.00 (reference), 1.10 (95\% CI 0.88, 1.38), 0.98 (95\% CI 0.77, 1.23), 1.01 (95\% CI $0 \cdot 80,1 \cdot 26)$ and 1.14 (95\% CI $0.91,1.43)$, for the lowest to highest quintiles, respectively $\left(P_{\text {trend }}=0 \cdot 40\right)$. 
Table 4. Quintiles $(\mathrm{Q})$ of total, processed and unprocessed red meat intakes and non-fatal acute myocardial infarction among 3130 men from the Costa Rica Heart Study

(Odds ratios and $95 \%$ confidence intervals)

\begin{tabular}{|c|c|c|c|c|c|c|c|c|c|c|}
\hline & \multirow[b]{2}{*}{ Q1 } & \multicolumn{2}{|c|}{ Q2 } & \multicolumn{2}{|c|}{ Q3 } & \multicolumn{2}{|c|}{ Q4 } & \multicolumn{2}{|c|}{ Q5 } & \multirow[b]{2}{*}{$P_{\text {trend }}$} \\
\hline & & OR & $95 \% \mathrm{Cl}$ & OR & $95 \% \mathrm{Cl}$ & OR & $95 \% \mathrm{Cl}$ & OR & $95 \% \mathrm{Cl}$ & \\
\hline \multicolumn{11}{|l|}{ Total red meat } \\
\hline Basic model* & 1.00 & 0.76 & $0.60,0.95$ & 0.80 & $0.64,1.02$ & 0.82 & $0.65,1.02$ & 1.29 & $1.03,1.61$ & 0.002 \\
\hline Multivariate $1 \dagger$ & 1.00 & 0.79 & $0.61,1.02$ & 0.78 & $0.60,1.01$ & 0.82 & $0.63,1.06$ & $1 \cdot 18$ & $0.92,1.52$ & 0.06 \\
\hline Multivariate $2 \ddagger$ & 1.00 & 0.92 & $0.70,1 \cdot 21$ & 0.98 & $0.74,1.30$ & 0.92 & $0.71,1.21$ & $1 \cdot 19$ & $0.92,1.55$ & 0.15 \\
\hline \multicolumn{11}{|c|}{ Processed red meat } \\
\hline Basic model* & 1.00 & 0.73 & $0.58,0.91$ & 0.70 & $0.56,0.88$ & 0.80 & $0.63,1.00$ & $1 \cdot 11$ & $0.88,1.39$ & 0.06 \\
\hline Multivariate $1 \dagger$ & 1.00 & 0.76 & $0.59,0.98$ & 0.73 & $0.56,0.95$ & 0.76 & $0.58,0.98$ & 1.07 & $0.83,1.38$ & 0.26 \\
\hline Multivariate $2 \ddagger$ & 1.00 & 0.91 & $0.69,1 \cdot 20$ & 1.00 & $0.75,1.34$ & 1.01 & $0.76,1.34$ & 1.25 & $0.96,1.65$ & 0.04 \\
\hline Multivariate $3 \S$ & 1.00 & 0.91 & $0.69,1 \cdot 20$ & 1.00 & $0.75,1.34$ & 1.01 & $0.76,1.35$ & 1.25 & $0.95,1.65$ & 0.04 \\
\hline \multicolumn{11}{|c|}{ Unprocessed red meat } \\
\hline Basic model ${ }^{*}$ & 1.00 & 0.96 & $0.76,1.20$ & 0.82 & $0.65,1.03$ & 0.98 & $0 \cdot 79,1.23$ & $1 \cdot 14$ & $0.92,1.43$ & $0 \cdot 13$ \\
\hline Multivariate $1 \dagger$ & 1.00 & 0.93 & $0.72,1 \cdot 20$ & 0.79 & $0.61,1.02$ & 0.95 & $0.74,1.21$ & 1.05 & $0.82,1.35$ & 0.52 \\
\hline Multivariate $2 \ddagger$ & 1.00 & $1 \cdot 15$ & $0.87,1.50$ & 0.94 & $0.72,1.24$ & 1.01 & $0.77,1.31$ & 1.05 & $0.81,1.36$ & 0.97 \\
\hline Multivariate $3 \|$ & 1.00 & $1 \cdot 12$ & $0.85,1.48$ & 0.93 & $0.71,1.23$ & 0.98 & $0.75,1.28$ & 1.01 & $0.78,1.32$ & 0.81 \\
\hline
\end{tabular}

* Adjusted for the matching factors (age and area of residence).

$\dagger$ In addition to the basic model, adjusted for waist:hip ratio (quintiles), physical activity level (quintiles), monthly household income (quintiles plus an indicator for missing data), history of diabetes (yes or no), history of hypertension (yes or no), smoking status (never, former or current $<10,10-20$ or $>20$ cigarettes/d) and alcohol intake (never, former or current tertiles).

$\ddagger$ In addition to the multivariate 1 , adjusted for total energy intake, fruit servings, green leafy vegetable servings, energy-adjusted chicken intake and energy-adjusted fish intake (all in quintiles).

$\S$ In addition to the multivariate 2, adjusted for energy-adjusted unprocessed red meat intake in quintiles (mutual adjustment)

|| In addition to the multivariate 2, adjusted for energy-adjusted processed red meat intake in quintiles (mutual adjustment).

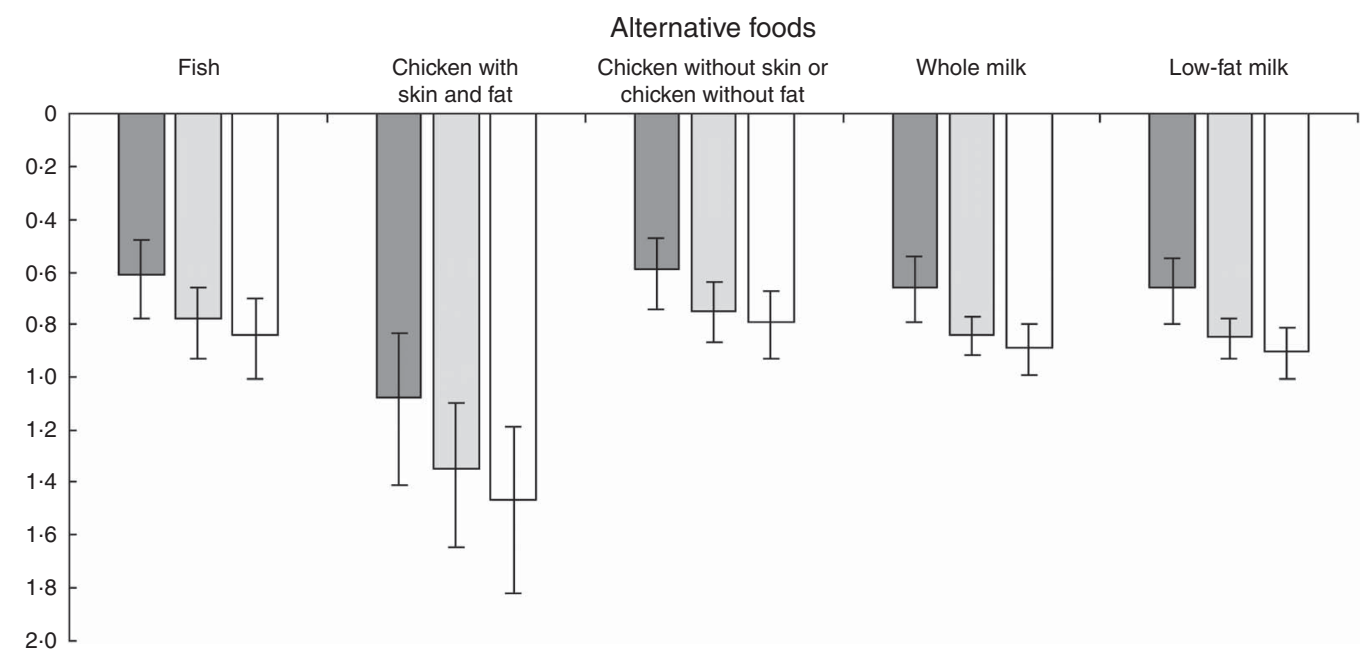

Fig. 2. Non-fatal acute myocardial infarction associated with substituting $50 \mathrm{~g}$ of alternative sources of protein for $50 \mathrm{~g}$ of processed ( $\square$ ), total ( $\square$ ) and unprocessed ( $\square$ ) red meat, based on the 2131 case-control pairs from the Costa Rica Heart Study. Values are OR and $95 \% \mathrm{Cl}$ estimated by including the numbers of servings (standardised to $50 \mathrm{~g}$ ) of both foods as continuous variables in the same model, which was also adjusted for waist:hip ratio (quintiles), physical activity level (quintiles), household income (quintiles plus an indicator for missing data), history of diabetes (yes or no), history of hypertension (yes or no), smoking status (never, former or current $<10,10-20$ or $>20$ cigarettes/d), alcohol intake (never, past or current tertiles) and total energy intake (quintiles). Low-fat milk includes 1 and $2 \%$ milk.

Substitutions of $50 \mathrm{~g}$ of fish, chicken without skin or chicken without fat, whole milk and low-fat milk for $50 \mathrm{~g}$ of red meat were associated with lower odds of acute MI, especially for total and processed red meat intakes (Fig. 2). For total red meat, the odds were $22 \%$ lower when replaced with fish (OR 0.78; $95 \%$ CI $0 \cdot 66,0 \cdot 93), 25 \%$ lower when replaced with chicken without skin or chicken without fat (OR 0.75; 95\% CI 0.64, 0.87), 16\% lower when replaced with whole milk (OR 0.84; 95\% CI 0.77, $0 \cdot 92$ ) and $15 \%$ lower when replaced with low-fat milk (OR 0.85; $95 \%$ CI $0.78,0.93)$. The odds were $35 \%$ higher when replacing total red meat with chicken with skin and fat (OR 1.35; $95 \%$ CI
$1 \cdot 10,1 \cdot 65)$. For processed red meat, the odds were $39 \%$ lower when replaced with fish (OR $0 \cdot 61 ; 95 \%$ CI $0 \cdot 48,0 \cdot 78$ ), $41 \%$ lower when replaced with chicken without skin or chicken without fat (OR 0.59; $95 \%$ CI 0.47, 0.74), 34\% lower when replaced with whole milk (OR 0.66; $95 \%$ CI $0.54,0.79$ ) and $34 \%$ lower when replaced with low-fat milk (OR 0.66; $95 \%$ CI 0.55, $0 \cdot 80$ ). Replacing processed red meat with chicken with skin and fat was not associated with the odds of acute MI (OR 1.08; $95 \%$ CI $0 \cdot 83,1 \cdot 41$ ). For unprocessed red meat, the corresponding OR associated with the replacements were 0.84 (95\% CI 0.70, 1.01) for fish, 1.47 (95\% CI 1.19, 1.82) for chicken with skin and fat, 
0.79 (95\% CI $0.67,0.93$ ) for chicken without skin or chicken without fat, 0.89 (95\% CI $0.80,0.99)$ for whole milk and 0.90 (95\% CI $0 \cdot 81,1 \cdot 01)$ for low-fat milk.

The estimates were fairly similar after excluding 621 participants who believed that diet was the main cause of MI (online Supplementary Table S3). Results were essentially the same with multiple imputations on missing income and did not appreciably change after excluding 315 participants with missing income or 529 participants with implausible total energy intake (data not shown).

\section{Discussion}

Results from this population-based case-control study in the Central Valley of Costa Rica suggest that intake of red meat, especially processed red meat, is associated with increased odds of non-fatal acute MI. Individuals who consume 1 serving/d of total red meat have a $31 \%$ higher odds of developing acute MI compared with those who consume 1.5 servings/week. Individuals who consume 5 servings/week of processed red meat have a $29 \%$ higher odds of acute MI compared with those who do not regularly consume processed red meat. We did not find an association between unprocessed red meat intake and the odds of acute MI. The sex-stratified analysis shows that the associations are stronger in women than in men.

To the best of our knowledge, this was the first study to evaluate the association between total, processed and unprocessed red meat intakes and acute MI in a Hispanic/Latino population undergoing a nutrition transition towards modernised dietary patterns. Because of the retrospective nature of our study, only survivors from acute MI were included as cases. Red meat intake is inversely associated with CVD survival in some populations $^{(4,8,9)}$, so it is possible that red meat intake was underestimated for cases in our study, which suggests that our study may have underestimated the potential adverse effects of red meat intake on acute MI.

Compared with other studies, most of the ones that found a positive association between red meat intake and CVD were conducted in developed Western countries, including the $\mathrm{USA}^{(8,9,27)}$, and some European countries such as France ${ }^{(28)}$ and Sweden ${ }^{(29)}$. Evidence from those countries indicates that processed red meat intake is associated with increased CVD incidence and mortality, whereas unprocessed red meat intake has weaker or no association with CVD. Associations found in our study are similar to those observed in developed Western countries, which may be partially due to the nutrition transition that Costa Rica has been experiencing ${ }^{(11)}$. In contrast, results from Asian countries, where red meat consumption is substantially lower than that in the Western countries, did not find a similar positive association between red meat intake and CVD $^{(30-32)}$. The null association observed in Asian countries is likely due to the restricted exposure distribution due to the generally low level of red meat consumption. For example, the highest category of processed red meat intake from studies in Europe and the USA ranges between 1 and 3 servings $/ \mathrm{d}^{(8-10)}$, whereas the highest level of processed red meat intake in the Japanese study is comparable to 4 servings/week $^{(30)}$. The medians of total red meat intake by quintiles in our study were very close to those in the Health Professionals Follow-up Study (1986-2008) ${ }^{(8)}$, and slightly lower than but generally comparable to those in the Nurses' Health Study (1980-2006) ${ }^{(25)}$. The specific type of red meat consumed (e.g. beef $v$. pork) and other effect modifiers such as genetics and cooking practices may also account for the different associations observed across populations. Substituting the same portion ( $50 \mathrm{~g}$ ) of fish, chicken (without skin or fat) and milk (high-fat and low-fat) is associated with 34-41\% lower odds of acute MI for processed red meat, and 15-25\% lower odds for total red meat. Substitutions of those alternative sources of protein for unprocessed red meat were also inversely associated with the odds of acute MI, although to a lesser extent than total and processed red meat. The substitution analysis confirms that the association between red meat intake and acute MI is driven largely by processed red meat.

Several nutrients and chemical compounds have been hypothesised to mediate the effects of red meat intake on CVD. Previous epidemiological studies have shown that high systemic Fe levels were associated with increased risk of acute $\mathrm{MI}^{(33-36)}$. Animal models also found that haem Fe could increase the risk of Fe toxicity, which might promote atherosclerosis through oxidative stress and endothelial dysfunction, and cause contractile dysfunction of the heart ${ }^{(37)}$. High dietary $\mathrm{Na}$ intake has been found associated with elevated blood pressure $^{(38)}$ and increased risk of cardiovascular events ${ }^{(39-41)}$ in several prospective cohort studies. Previous studies had inconsistent and even opposite conclusions regarding the association between dietary nitrate and $\mathrm{CVD}^{(42-44)}$. Many other potential mediators, such as phosphate ${ }^{(45)}$ and polycyclic aromatic hydrocarbon ${ }^{(46)}$, may also be at play.

The observed association between red meat intake and acute MI was stronger in women than in men, especially for unprocessed red meat intake. Limited evidence exists regarding sex as a potential modifier of the effect of red meat intake on CVD. A previous sex-stratified meta-analysis of cohort studies found that the association between unprocessed and processed red meat intake and CVD mortality was significant in women but not in $\operatorname{men}^{(4)}$. It is unclear whether the potentially heterogeneous results by sex in our study are due to true physiological differences between the sexes or simply due to the larger random error among women (only $27 \%$ of the study sample were women). The results were essentially the same when the history of hypertension was not adjusted for in the models, which either suggests that hypertension status did not considerably confound the association when other covariates were adjusted for, or that hypertension status may not be a mediator of the association. However, due to the retrospective design of the study, we were unable to ascertain the causal role of hypertension status.

The population-based design is a strength of our study. The comprehensive social services in Costa Rica ensured that all persons living in the catchment area had access to medical care regardless of their socioeconomic status. Therefore, control subjects were unlikely to have had undiagnosed CVD because of limited access to health care. With the help of the National Census and Statistics Bureau of Costa Rica, we were able to select controls directly from the source population that gave rise to the study cases. Thus, selection bias was highly unlikely 
because sampling of controls was independent of the exposure status. The Central Valley of Costa Rica included diverse lifestyle and socioeconomic characteristics, so the results are also likely to be generalisable to the target population of Hispanics/Latinos in Costa Rica.

This study also has several potential limitations. First, the retrospective FFQ was prone to more recall bias than prospective dietary measurements because responses from the cases might be modified by the knowledge of their acute MI (i.e. differential exposure measurement error). However, between 1994 and 2004, during the nutrition transition in Costa Rica, red meat intake was highly unlikely to be considered by the general population as a risk factor for CVD. Only 621 participants $(14.6 \%$ of the study sample) believed that diet was the main cause of MI, and the estimates were essentially the same after excluding those participants. Even if recall bias were indeed present, it would have affected processed and unprocessed red meat intakes equivalently. Thus, the lack of association between unprocessed red meat and acute MI provided further evidence that the extent of recall bias was not considerable. Still, due to the retrospective nature of our study, recall bias could not be completely ruled out, and the results must be interpreted with caution. Second, self-reported FFQ usually have more measurement error than 24-h recalls and diet records ${ }^{(47)}$. However, FFQ are better at measuring long-term intake, and the FFQ used in this study was developed and validated specifically for the Costa Rican population ${ }^{(16,17)}$, so measurement error was minimised. Third, although we adjusted for several covariates, residual confounding could still be present as a result of imperfect covariate measurement, or failure to measure or adjust for other important covariates, as in all observational studies.

In conclusion, our study found that red meat intake, especially processed red meat intake, is associated with higher odds of non-fatal acute MI in a Hispanic/Latino population from the Costa Rica Hear Study. The positive association is particularly strong when total red meat intake exceeds $110 \mathrm{~g} / \mathrm{d}$ ( 1 serving/d) or when processed red meat intake exceeds $36 \mathrm{~g} / \mathrm{d}$ (5 servings/ week). The associations between total, processed and unprocessed red meat intake and acute MI are stronger in women than in men. The potential adverse effect of red meat intake on CVD in developing Hispanic/Latino countries is of great concern as it is projected that red meat consumption will continue to increase in those countries ${ }^{(1-3)}$. Based on our results, we further hypothesise that the increased CVD incidence in Costa Rica and other developing Hispanic/Latino populations could partially be attributed to the increased consumption of red meat resulting from the nutrition transition. Future studies with prospective and longitudinal measures of dietary intake are warranted in developing Hispanic/Latino countries that are currently transitioning to a Westernised diet.

\section{Acknowledgements}

The authors are grateful to the study participants and to the staff of Proyecto Salud Coronaria, San José, Costa Rica. This work was supported by the National Institutes of Health (HL49086, HL60692), USA. The National Institutes of Health had no role in the design, analysis or writing of this article.
The authors' contributions were as follows: H. C. and A. B. designed the study and collected data; D. W. carried out data analysis; D. W., H. C. and A. B. wrote the manuscript. All authors read and approved the final version of the manuscript.

None of the authors has any conflicts of interest to declare.

\section{Supplementary material}

For supplementary material/s referred to in this article, please visit https://doi.org/10.1017/S000711451700201X

\section{References}

1. Popkin BM, Adair LS \& Ng SW (2012) Global nutrition transition and the pandemic of obesity in developing countries. Nutr Rev 70, 3-21.

2. Bruinsma J (2003) World Agriculture: Towards 2015/2030: An FAO perspective. London: Earthscan.

3. Food and Agriculture Organization of the United Nations \& Organisation for Economic Co-operation and Development (2014) OECD-FAO Agricultural Outlook 2014-2023, 20th ed. Paris: OECD.

4. Abete I, Romaguera D, Vieira AR, et al. (2014) Association between total, processed, red and white meat consumption and all-cause, CVD and IHD mortality: a meta-analysis of cohort studies. Br J Nutr 112, 762-775.

5. Celermajer DS, Chow CK, Marijon E, et al. (2012) Cardiovascular disease in the developing world: prevalences, patterns, and the potential of early disease detection. J Am Coll Cardiol 60, 1207-1216.

6. Reddy KS (2004) Cardiovascular disease in non-Western countries. N Engl J Med 350, 2438-2440.

7. Wolk A (2016) Potential health hazards of eating red meat. J Intern Med 281, 106-122.

8. Pan A, Sun Q, Bernstein AM, et al. (2012) Red meat consumption and mortality: results from 2 prospective cohort studies. Arch Intern Med 172, 555-563.

9. Sinha R, Cross AJ, Graubard BI, et al. (2009) Meat intake and mortality: a prospective study of over half a million people. Arch Intern Med 169, 562-571.

10. Rohrmann S, Overvad K, Bueno-de-Mesquita HB, et al. (2013) Meat consumption and mortality - results from the European Prospective Investigation into Cancer and Nutrition. BMC Med 11, 63.

11. Araya MR \& Padilla SG (2004) Trends in mortality from ischemic heart disease and acute myocardial infarction in Costa Rica, 1970-2001. Rev Panam Salud Publica 16, 295-301.

12. World Health Organization (2014) Noncommunicable Diseases Country Profiles 2014. Geneva: WHO.

13. Kabagambe EK, Baylin A, Ruiz-Narvarez E, et al. (2005) Decreased consumption of dried mature beans is positively associated with urbanization and nonfatal acute myocardial infarction. J Nutr 135, 1770-1775.

14. Tunstall-Pedoe H, Kuulasmaa K, Amouyel P, et al. (1994) Myocardial infarction and coronary deaths in the World Health Organization MONICA Project. Registration procedures, event rates, and case-fatality rates in 38 populations from 21 countries in four continents. Circulation 90, 583-612.

15. Campos H \& Siles X (2000) Siesta and the risk of coronary heart disease: results from a population-based, case-control study in Costa Rica. Int J Epidemiol 29, 429-437.

16. Kabagambe EK, Baylin A, Allan DA, et al. (2001) Application of the method of triads to evaluate the performance of food 
frequency questionnaires and biomarkers as indicators of long-term dietary intake. Am J Epidemiol 154, 1126-1135.

17. Baylin A, Kabagambe EK, Siles X, et al. (2002) Adipose tissue biomarkers of fatty acid intake. Am J Clin Nutr $\mathbf{7 6}$, 750-757.

18. Willett WC, Sampson L, Stampfer MJ, et al. (1985) Reproducibility and validity of a semiquantitative food frequency questionnaire. Am J Epidemiol 122, 51-65.

19. Campos H, Willett WC, Peterson RM, et al. (1991) Nutrient intake comparisons between Framingham and rural and Urban Puriscal, Costa Rica. Associations with lipoproteins, apolipoproteins, and low density lipoprotein particle size. Arterioscler Thromb 11, 1089-1099.

20. US Department of Agriculture, Agricultural Research Service (1999) Composition of foods: raw, processed and prepared. USDA nutrient database for standard reference, release 13, pp. 1-37. Beltsville, MD: USDA, Agricultural Research Service, Beltsville Human Nutrition Research Center, Nutrient Data Laboratory.

21. Micha R, Wallace SK \& Mozaffarian D (2010) Red and processed meat consumption and risk of incident coronary heart disease, stroke, and diabetes mellitus: a systematic review and meta-analysis. Circulation 121, 2271-2283.

22. Chen GC, Lv DB, Pang Z, et al. (2013) Red and processed meat consumption and risk of stroke: a meta-analysis of prospective cohort studies. Eur J Clin Nutr 67, 91-95.

23. Micha R, Michas G \& Mozaffarian D (2012) Unprocessed red and processed meats and risk of coronary artery disease and type 2 diabetes - an updated review of the evidence. Curr Atheroscler Rep 14, 515-524.

24. Willett WC, Howe GR \& Kushi LH (1997) Adjustment for total energy intake in epidemiologic studies. Am J Clin Nutr $\mathbf{6 5}$, 1220S-1228S

25. Bernstein AM, Sun Q, Hu FB, et al. (2010) Major dietary protein sources and risk of coronary heart disease in women. Circulation 122, 876-883.

26. Berglund PA \& Heeringa S (2014) Multiple Imputation of Missing Data Using SAS. Cary, NC: SAS Institute.

27. Ashaye A, Gaziano J \& Djousse L (2011) Red meat consumption and risk of heart failure in male physicians. Nutr Metab Cardiovasc Dis 21, 941-946.

28. Lajous M, Bijon A, Fagherazzi G, et al. (2014) Processed and unprocessed red meat consumption and hypertension in women. Am J Clin Nutr 100, 948-952.

29. Kaluza J, Akesson A \& Wolk A (2014) Processed and unprocessed red meat consumption and risk of heart failure: prospective study of men. Circ Heart Fail 7, 552-557.

30. Nagao M, Iso H, Yamagishi K, et al. (2012) Meat consumption in relation to mortality from cardiovascular disease among Japanese men and women. Eur J Clin Nutr 66, 687-693.
31. Lee JE, McLerran DF, Rolland B, et al. (2013) Meat intake and cause-specific mortality: a pooled analysis of Asian prospective cohort studies. Am J Clin Nutr 98, 1032-1041.

32. Takata Y, Shu XO, Gao YT, et al. (2013) Red meat and poultry intakes and risk of total and cause-specific mortality: results from cohort studies of Chinese adults in Shanghai. PLOS ONE 8, e56963.

33. Salonen JT, Nyyssonen K, Korpela H, et al. (1992) High stored iron levels are associated with excess risk of myocardial infarction in eastern Finnish men. Circulation 86, 803-811.

34. Morrison HI, Semenciw RM, Mao Y, et al. (1994) Serum iron and risk of fatal acute myocardial infarction. Epidemiology 5, 243-246.

35. Tuomainen TP, Punnonen K, Nyyssonen K, et al. (1998) Association between body iron stores and the risk of acute myocardial infarction in men. Circulation 97, 1461-1466.

36. Holay MP, Choudhary AA \& Suryawanshi SD (2012) Serum ferritin-a novel risk factor in acute myocardial infarction. Indian Heart J 64, 173-177.

37. Vinchi F, Muckenthaler MU, Da Silva MC, et al. (2014) Atherogenesis and iron: from epidemiology to cellular level. Front Pharmacol 5, 94.

38. Mente A, O'Donnell MJ, Rangarajan S, et al. (2014) Association of urinary sodium and potassium excretion with blood pressure. $N$ Engl J Med 371, 601-611.

39. Alderman MH \& Cohen HW (2012) Dietary sodium intake and cardiovascular mortality: controversy resolved? Curr Hypertens Rep 14, 193-201.

40. O'Donnell MJ, Yusuf S, Mente A, et al. (2011) Urinary sodium and potassium excretion and risk of cardiovascular events. JAMA 306, 2229-2238.

41. O'Donnell M, Mente A, Rangarajan S, et al. (2014) Urinary sodium and potassium excretion, mortality, and cardiovascular events. $N$ Engl J Med 371, 612-623.

42. Machha A \& Schechter AN (2011) Dietary nitrite and nitrate: a review of potential mechanisms of cardiovascular benefits. Eur J Nutr 50, 293-303.

43. Hord NG (2011) Dietary nitrates, nitrites, and cardiovascular disease. Curr Atheroscler Rep 13, 484-492.

44. Adamson RH \& Thorgeirsson UP (1995) Carcinogens in foods: heterocyclic amines and cancer and heart disease. Adv Exp Med Biol 369, 211-220.

45. Tonelli M, Sacks F, Pfeffer M, et al. (2005) Relation between serum phosphate level and cardiovascular event rate in people with coronary disease. Circulation 112, 2627-2633.

46. Xu X, Cook RL, Ilacqua VA, et al. (2010) Studying associations between urinary metabolites of polycyclic aromatic hydrocarbons (PAHs) and cardiovascular diseases in the United States. Sci Total Environ 408, 4943-4948.

47. Garrow JS (1995) Validation of methods for estimating habitual diet: proposed guidelines. Eur J Clin Nutr 49, 231-232. 